\title{
A Modified Techniques of Fractional-Order Cauchy-Reaction Diffusion Equation via Shehu Transform
}

\author{
Mounirah Areshi $\mathbb{D}^{1},{ }^{1}$ A. M. Zidan $\mathbb{D}^{2},{ }^{2,3}$ Rasool Shah $\mathbb{D}^{4},{ }^{4}$ and Kamsing Nonlaopon $\mathbb{D}^{5}$ \\ ${ }^{1}$ Department of Mathematics, Faculty of Science, University of Tabuk, Tabuk 71491, Saudi Arabia \\ ${ }^{2}$ Department of Mathematics, College of Science, King Khalid University, P.O. Box: 9004, Abha 61413, Saudi Arabia \\ ${ }^{3}$ Department of Mathematics, Faculty of Science, Al-Azhar University, Assuit 71511, Egypt \\ ${ }^{4}$ Department of Mathematics, Abdul Wali Khan University Mardan 23200, Pakistan \\ ${ }^{5}$ Department of Mathematics, Faculty of Science, Khon Kaen University, Khon Kaen 40002, Thailand
}

Correspondence should be addressed to Kamsing Nonlaopon; nkamsi@kku.ac.th

Received 9 May 2021; Revised 29 May 2021; Accepted 8 July 2021; Published 26 July 2021

Academic Editor: Nehad Ali Shah

Copyright (c) 2021 Mounirah Areshi et al. This is an open access article distributed under the Creative Commons Attribution License, which permits unrestricted use, distribution, and reproduction in any medium, provided the original work is properly cited.

\begin{abstract}
In this article, the iterative transformation method and homotopy perturbation transformation method are applied to calculate the solution of time-fractional Cauchy-reaction diffusion equations. In this technique, Shehu transformation is combined of the iteration and the homotopy perturbation techniques. Four examples are examined to show validation and the efficacy of the present methods. The approximate solutions achieved by the suggested methods indicate that the approach is easy to apply to the given problems. Moreover, the solution in series form has the desire rate of convergence and provides closed-form solutions. It is noted that the procedure can be modified in other directions of fractional order problems. These solutions show that the current technique is very straightforward and helpful to perform in applied sciences.
\end{abstract}

\section{Introduction}

In recent centuries, fractional partial differential equation (FPDE) fundamental signification is well-known in different engineering fields. Numerous physical phenomena can be modelled using FPDEs in various engineering and science fields such as physics, finance, aerospace, mechanics, biology, signal processing, biochemistry, and polymers $[1,2]$. It is challenging to create computational models for any natural phenomenon using a traditional differential operator since it can describe those phenomena, primarily hereditary properties. They have a memory effect and the integrated capacity to describe and explain physical phenomena that cannot be adequately explained using an integer-order differential equation. The nonlocality of fractional operators in partial differential equations is one purpose for their importance in modeling different areas of chemical, biological, psychological, thermoplasticity, physical, and mechanical systems [3-5].
Reaction-diffusion (RD) equations define various nonlinear schemes in chemistry, physics, biology, ecology, and other field sciences. RD equations are commonly used in ecology as a model for geographic impacts. They agree on three significant forms of environmental concepts: a minimum patch size required to maintain a population, the establishment of spatial trends in population distributions in homogeneous ecosystems, and the propagation of wavefronts associated with biological invasions. $\mathrm{RD}$ equations can be studied using techniques from the concept of PDEs and dynamical schemes [6-9].

The equation of reaction-diffusion write of the following form:

$$
\frac{\partial \mu}{\partial \eta}=\Delta \mu+g(\mu, \nabla \mu: \varphi, \eta) .
$$

The term of $\Delta \mu$ is diffusion term and $g(\mu, \nabla \mu: \varphi, \eta)$ is the reaction function. Moreover, the diffusion general term is 
$A(\mu)$, where $A$ is a 2 nd order nonlinear elliptic operators. In this article, we consider the fractional-order one-dimensional, reaction-diffusion equation

$$
\frac{\partial^{\delta} \mu(\varphi, \eta)}{\partial \eta^{\delta}}=D \frac{\partial^{2} \mu(\varphi, \eta)}{\partial \varphi^{2}}+r(\varphi, \eta) \mu(\varphi, \eta),(\varphi, \eta) \in \Omega \subset R^{2}
$$

If $\delta=1$, then, it becomes classical reaction-diffusion equation, where $\mu$ is the concentration, $r$ is the reaction parameter, and $D>0$ is the diffusion coefficient, with the initial and boundaries conditions

$$
\begin{aligned}
& \mu(\varphi, 0)=f(\varphi), \varphi \in R, \\
& \mu(0, \eta)=g_{0}(\eta) \cdot \frac{\partial \mu}{\partial \varphi}(0, \eta)=g_{1}(t), \eta \in R .
\end{aligned}
$$

The model defined by equations (2) and (3) is called the characteristic Cauchy model in the domain $\Omega=R \times R_{+}$, and the model given by equations (2) and (4) is called the noncharacteristic Cauchy equation in the domain $\Omega=R_{+} \times R$, using different analytical and numerical methods to solve reaction diffusion equation, such as Picard technique [10], homotopy perturbation technique [3], differential transformation technique and variation iteration technique [11], Adomian decomposition method [12], homotopy analysis method [13], fractional iteration algorithm I [14], new Sumudu transform iterative method [15], and finitedifference discretization scheme [16].

Daftardar-Gejji and Jafari in 2006 [17] suggested DJM solves linear and nonlinear differential equations. The DJM is simple and easy to comprehend and use, and it provides faster numerical solutions than the variational iterative technique [18] and the Adomian decomposition technique $[19,20]$. The new iterative transformation method (NITM) is a combination of Shehu transform and the new iterative approach that provides the solution in the form of convergent series in an easy way. Another technique is the homotopy perturbation transform method (HPTM) to some applicable fractional models arising in real-life problems. The HPTM is applied directly to fractional models without any linearization, discretization, or variable transforms. The HPTM is an iterative technique that converges to solutions in closed form or approximate solutions. The nonlinear terms are decomposed successfully via He's polynomials, and the fractional derivatives are computed in the Caputo sense. Applications of three fractional models are demonstrated, and the analytical and numerical simulations of the three fractional models are provided to bolster the efficiency, simplicity, and high accuracy of the HPTM. Many researchers use this method to solve different fractionalorder partial differential equations, such as fractional order gas dynamic equation [21], convection-diffusion problems [22], and Klein-Gordon equations [23].

This paper uses the Iterative transform method and homotopy perturbation transform method to solve fractional Cauchy-reaction diffusion equation equations using the frac- tional operator of Caputo type. The fractional calculus fundamental definitions are defined in Section 2, writing the general methodologies in Sections 3 and 4, many test models show the effectiveness of suggested techniques in Sections 5 and 6. Finally, the conclusion is given in Section 7.

\section{Basic Definitions}

2.1. Definition. The Riemann fractional integral is given as $[24,25]$

$$
I_{0}^{\delta} h(\eta)=\frac{1}{\Gamma(\delta)} \int_{0}^{\eta}(\eta-s)^{\delta-1} h(s) d s
$$

2.2. Definition. The Caputo fractional derivative of $f(\eta)$ is defined as $[24,25]$

$$
\begin{gathered}
D_{\eta}^{\delta} f(\eta)=I^{j-\delta} f^{j}, j-1<\delta<j, j \in \mathbb{N}, \\
\frac{d^{j}}{d \eta^{j}} h(\eta), \delta=j, j \in \mathbb{N} .
\end{gathered}
$$

2.3. Definition. The integral transform of Shehu transform in Set A, the function is defined by [26-28]

$$
A=\left\{\mu(\eta): \exists, \rho_{1}, \rho_{2}>0,|\mu(\eta)|<M e^{\frac{|\eta|}{\rho_{i}}} \text { if } \eta \in[0, \infty)\right.
$$

The transformation of Shehu is defined as $S($.$) for a$ function $\mu(\eta)$ is given as

$$
S\{\mu(\eta)\}=V(s, v)=\int_{0}^{\infty} e^{\frac{-s \eta}{v}} \mu(\eta) d \eta, \eta>0, s>0 .
$$

The Shehu transform of a function $\mu(\eta)$ is $V(s, v)$ : then, $\mu(\eta)$ is called the inverse of $V(s, v)$ which is given as

$$
\begin{aligned}
S^{-1}\{V(s, v)\}= & \mu(\eta), \text { for } \eta \geq 0, S^{-1} \text { is the Shehu } \\
& \text { inverse transformation. }
\end{aligned}
$$

2.4. Definition. The $n$th derivative of Shehu transformation is defined as [27-29]

$$
S\left\{\mu^{(j)}(\eta)\right\}=\frac{s^{j}}{v^{j}} V(s, v)-\sum_{k=0}^{j-1}\left(\frac{s}{v}\right)^{j-k-1} \mu^{(k)}(0) .
$$

2.5. Definition. The Shehu transform of fractional derivative is given as [26-28].

$$
S\left\{\mu^{(\delta)}(\eta)\right\}=\frac{s^{\delta}}{v^{\delta}} \mu(s, v)-\sum_{k=0}^{j-1}\left(\frac{s}{v}\right)^{\delta-k-1} \mu^{(k)}(0), 0<\delta \leq n .
$$




\section{The HPTM General Implementation}

Consider the general fractional partial differential equation solve to HPTM:

$$
\begin{aligned}
& D_{\eta}^{\delta} \mu(\varphi, \eta)+M \mu(\varphi, \eta)+N \mu(\varphi, \eta) \\
& \quad=h(\varphi, \eta), \eta>0,0<\delta \leq 1, \mu(\varphi, 0) \\
& \quad=g(\varphi), \mu \in \Re .
\end{aligned}
$$

Applying Shehu transformation of equation (13), we get

$$
\begin{aligned}
S[ & \left.D_{\eta}^{\delta} \mu(\varphi, \eta)+M \mu(\varphi, \eta)+N \mu(\varphi, \eta)\right] \\
& =S[h(\varphi, \eta)], \eta>0,0<\delta \leq 1, \mu(\varphi, \eta) \\
& =\frac{v}{s} g(\varphi)+\frac{v^{\delta}}{s^{\delta}} S[h(\varphi, \eta)]-\frac{v^{\delta}}{s^{\delta}} S[M \mu(\varphi, \eta)+N \mu(\varphi, \eta)] .
\end{aligned}
$$

Now, using inverse Shehu transformation, we achieve as

$$
\mu(\varphi, \eta)=F(\varphi, \eta)-S^{-1}\left[\frac{v^{\delta}}{s^{\delta}} S\{M \mu(\varphi, \eta)+N \mu(\varphi, \eta)\}\right]
$$

where

$$
\begin{aligned}
F(\varphi, \eta) & =S^{-1}\left[\frac{v}{s} g(\varphi)+\frac{v^{\delta}}{s^{\delta}} S[h(\varphi, \eta)]\right] \\
& =g(\mu)+S^{-1}\left[\frac{v^{\delta}}{s^{\delta}} S[h(\varphi, \eta)]\right]
\end{aligned}
$$

The parameter $p$ show the producer of perturbation is given as

$$
\mu(\varphi, \eta)=\sum_{k=0}^{\infty} p^{k} \mu_{k}(\varphi, \eta)
$$

where the perturbation $p$ is parameter and $p \in[0,1]$.

The nonlinear components can be defined as

$$
N \mu(\varphi, \eta)=\sum_{k=0}^{\infty} p^{k} H_{k}\left(\mu_{k}\right)
$$

where He's polynomials $H_{n}$ in terms of $\mu_{0}, \mu_{1}, \mu_{2}, \cdots, \mu_{n}$, and can be calculated as

$$
H_{n}\left(\mu_{0}, \mu_{1}, \cdots, \mu_{n}\right)=\frac{1}{\delta(n+1)} D_{p}^{k}\left[N\left(\sum_{k=0}^{\infty} p^{k} \mu_{k}\right)\right]_{p=0},
$$

where $D_{p}^{k}=\partial^{k} / \partial^{k}$
Substituting equations (18) and (19) in equation (15), we obtain as

$$
\begin{aligned}
& \sum_{k=0}^{\infty} p^{k} \mu_{k}(\varphi, \eta) \\
& =F(\varphi, \eta)-p \\
& \quad \times\left[S^{-1}\left\{\frac{v^{\delta}}{s^{\delta}} S\left\{M \sum_{k=0}^{\infty} p^{k} \mu_{k}(\varphi, \eta)+\sum_{k=0}^{\infty} p^{k} H_{k}\left(\mu_{k}\right)\right\}\right\}\right]
\end{aligned}
$$

The $p$ coefficients comparison on both sides, we have

$$
\begin{aligned}
p^{0}: \mu_{0}(\varphi, \eta) & =F(\varphi, \eta), \\
p^{1}: \mu_{1}(\varphi, \eta) & =S^{-1}\left[\frac{v^{\delta}}{s^{\delta}} S\left(M \mu_{0}(\varphi, \eta)+H_{0}(\mu)\right)\right], \\
p^{2}: \mu_{2}(\varphi, \eta) & =S^{-1}\left[\frac{v^{\delta}}{s^{\delta}} S\left(M \mu_{1}(\varphi, \eta)+H_{1}(\mu)\right)\right], \\
\vdots & \\
p^{k}: \mu_{k}(\varphi, \eta) & =S^{-1}\left[\frac{v^{\delta}}{s^{\delta}} S\left(M \mu_{k-1}(\varphi, \eta)+H_{k-1}(\mu)\right)\right], k>0, k \in N .
\end{aligned}
$$

The $\mu_{k}(\varphi, \eta)$ components simply calculated to the convergence series form. We can achieve $p \longrightarrow 1$,

$$
\mu(\varphi, \eta)=\lim _{M \longrightarrow \infty} \sum_{k=1}^{M} \mu_{k}(\varphi, \eta)
$$

\section{The NITM General Implementation}

Consider the general fractional partial differential equation solve to NITM:

$D_{\eta}^{\delta} \mu(\varphi, \eta)+M \mu(\varphi, \eta)+N \mu(\varphi, \eta)=h(\varphi, \eta), n \in N, n-1<\delta \leq n$,

where linear and nonlinear functions represent by $M$ and $N$.

With initial condition

$$
\mu^{k}(\varphi, 0)=g_{k}(\varphi), k=0,1,2, \cdots, n-1
$$

Apply the Shehu transformation of equation (23), we obtain as

$$
S\left[D_{\eta}^{\delta} \mu(\varphi, \eta)\right]+S[N \mu(\varphi, \eta)+M \mu(\varphi, \eta)]=E[h(\varphi, \eta)]
$$


Using the differentiation property of Shehu transform is given as

$$
\begin{aligned}
S[\mu(\varphi, \eta)]= & \frac{v}{s} \mu(\varphi, 0)+\frac{v^{\delta}}{s^{\delta}} S[h(\varphi, \eta)] \\
& -\frac{v^{\delta}}{s^{\delta}} S[N \mu(\varphi, \eta)+M \mu(\varphi, \eta)],
\end{aligned}
$$
have

The Shehu inverse transform applies in equation (26), we

$$
\begin{aligned}
\mu(\varphi, \eta)= & S^{-1}\left[\left\{\frac{v}{s} \mu(\varphi, 0)+\frac{v^{\delta}}{s^{\delta}} S[h(\varphi, \eta)]\right\}\right] \\
& -S^{-1}\left[\frac{v^{\delta}}{s^{\delta}} S[N \mu(\varphi, \eta)+M \mu(\varphi, \eta)]\right] .
\end{aligned}
$$

In the iterative method, we get

$$
\begin{aligned}
\mu(\varphi, \eta) & =\sum_{m=0}^{\infty} \mu_{m}(\varphi, \eta) . \\
N\left(\sum_{m=0}^{\infty} \mu_{m}(\varphi, \eta)\right) & =\sum_{m=0}^{\infty} N\left[\mu_{m}(\varphi, \eta)\right],
\end{aligned}
$$

the nonlinear term $N$ is expressed as

$$
\begin{aligned}
N\left(\sum_{m=0}^{\infty} \mu_{m}(\varphi, \eta)\right)= & \mu_{0}(\varphi, \eta)+N\left(\sum_{k=0}^{m} \mu_{k}(\varphi, \eta)\right) \\
& -M\left(\sum_{k=0}^{m} \mu_{k}(\varphi, \eta)\right) .
\end{aligned}
$$

Substituting equations (28), (29), and (30) in equation (27), we can obtain the solution

$$
\begin{aligned}
\sum_{m=0}^{\infty} \mu_{m}(\varphi, \eta) & \\
=S^{-1} & {\left[\frac{v^{\delta}}{s^{\delta}}\left(\sum_{k=0}^{m} s^{2-\varphi+k} u^{k}(\varphi, 0)+S[h(\varphi, \eta)]\right)\right] } \\
& -S^{-1}\left[\frac{v^{\delta}}{s^{\delta}}\left[N\left(\sum_{k=0}^{m} \mu_{k}(\varphi, \eta)\right)-M\left(\sum_{k=0}^{m} \mu_{k}(\varphi, \eta)\right)\right]\right] .
\end{aligned}
$$

The iterative method can be defined as

$$
\begin{aligned}
& \mu_{0}(\varphi, \eta)=S^{-1}\left[\frac{v^{\delta}}{s^{\delta}}\left(\sum_{k=0}^{m} s^{2-\varphi+k} u^{k}(\varphi, 0)+\frac{v^{\delta}}{s^{\delta}} E(g(\varphi, \eta))\right)\right], \\
& \mu_{1}(\varphi, \eta)=-S^{-1}\left[\frac { v ^ { \delta } } { s ^ { \delta } } S \left[N\left[\mu_{0}(\varphi, \eta)\right]+M\left[\mu_{0}(\varphi, \eta)\right],\right.\right.
\end{aligned}
$$

$$
\begin{aligned}
\mu_{m+1}(\varphi, \eta)= & -S^{-1}\left[\frac { v ^ { \delta } } { s ^ { \delta } } S \left[-N\left(\sum_{k=0}^{m} \mu_{k}(\varphi, \eta)\right)\right.\right. \\
& \left.-M\left(\sum_{k=0}^{m} \mu_{k}(\varphi, \eta)\right)\right], m \geq 1 .
\end{aligned}
$$

Finally, equations (23) and (24) provide the $m$-terms solution in series form is achieved as

$$
\begin{aligned}
\mu(\varphi, \eta) \cong & \mu_{0}(\varphi, \eta)+\mu_{1}(\varphi, \eta)+\mu_{2}(\varphi, \eta)+. . \cdots \\
& +\mu_{m}(\varphi, \eta), m=1,2, \cdots
\end{aligned}
$$

Similarly, the convergence proof of the NITM, see [17].

4.1. Example. Consider the fractional Cauchy-reaction diffusion equation is given as:

$$
D_{\eta}^{\delta} \mu(\varphi, \eta)=D_{\eta}^{2} \mu(\varphi, \eta)-\mu(\varphi, \eta), 0<\delta \leq 1,
$$

with the initial and boundaries conditions

$$
\begin{aligned}
\mu(\varphi, 0) & =e^{-\varphi}+\varphi=g(\varphi), \mu(0, \eta)=1=f_{0}(\eta), \\
\frac{\partial \mu(0, \eta)}{\partial \eta} & =e^{-\eta}-1=f_{1}(\eta), \varphi, \eta \in R .
\end{aligned}
$$

Applying Shehu transform in equation (36), we get

$$
S[\mu(\varphi, \eta)]=\frac{v}{s}\left(e^{-\varphi}+\varphi\right)+s^{\delta} S\left[D_{\eta}^{2} \mu-\mu\right] .
$$

Using inverse Shehu transform in the above equation, we get

$$
\mu(\varphi, \eta)=\left(e^{-\varphi}+\varphi\right)+S^{-1}\left(s^{\delta} S\left[D_{\eta}^{2} \mu-\mu\right]\right) .
$$

First, we implement the NITM, we get the following

$$
\begin{aligned}
& \mu_{0}(\varphi, \eta)=e^{-\varphi}+\varphi \\
& \mu_{1}(\varphi, \eta)=S^{-1}\left[s^{\delta} S\left\{D_{\eta}^{2} \mu_{0}-\mu_{0}\right\}\right]=\varphi \frac{\left(-\eta^{\delta}\right)}{\Gamma(\delta+1)}, \\
& \mu_{2}(\varphi, \eta)=S^{-1}\left[s^{\delta} S\left\{D_{\eta}^{2} \mu_{1}-\mu_{1}\right\}\right]=\varphi \frac{\left(-\eta^{\delta}\right)^{2}}{\Gamma(2 \delta+1)},
\end{aligned}
$$

$$
\mu_{3}(\varphi, \eta)=S^{-1}\left[s^{\delta} S\left\{D_{\eta}^{2} \mu_{2}-\mu_{2}\right\}\right]=\varphi \frac{\left(-\eta^{\delta}\right)^{3}}{\Gamma(3 \delta+1)},
$$

$$
\mu_{n}(\varphi, \eta)=S^{-1}\left[s^{\delta} S\left\{D_{\eta}^{2} \mu_{n}-\mu_{n}\right\}\right]=\varphi \frac{\left(-\eta^{\delta}\right)^{n}}{\Gamma(n \delta+1)}, n \geq 0
$$


The series form solution is given as

$\mu(\varphi, \eta)=\mu_{0}(\varphi, \eta)+\mu_{1}(\varphi, \eta)+\mu_{2}(\varphi, \eta)+\mu_{3}(\varphi, \eta)+\cdots \mu_{n}(\varphi, \eta)$.

The approximate solutions is achieved as

$$
\begin{aligned}
\mu(\varphi, \eta)= & e^{-\varphi}+\varphi\left\{1-\frac{\eta^{\delta}}{\Gamma(\delta+1)}+\frac{\eta^{2 \delta}}{\Gamma(2 \delta+1)}\right. \\
& \left.-\frac{\eta^{3 \delta}}{\Gamma(3 \delta+1)}+\cdots+\frac{\left(-\eta^{\delta}\right)^{n}}{\Gamma(n \delta+1)}\right\} .
\end{aligned}
$$

Now we implement the HPTM, we obtain as

$$
\sum_{n=0}^{\infty} p^{n} \mu_{n}(\varphi, \eta)=\left(e^{-\varphi}+\varphi\right)+p\left[S^{-1}\left\{s^{\delta} S\left(\sum_{n=0}^{\infty} p^{n} H_{n}(\mu)\right)\right\}\right]
$$

Where the nonlinear function of the polynomial is shown by $H_{n}(\mu)$. For instance, the He's polynomial component is obtained through the recursive relation $H_{n}(\mu)=D_{\eta}^{2} \mu_{n}-\mu_{n}$, $\forall n \in N$. Now, on both sides, the coefficient of corresponding power $p$ is calculated, and the following solution is obtained as follows:

$$
\begin{aligned}
p^{0}: \mu_{0}(\varphi, \eta) & =e^{-\varphi}+\varphi \\
p^{1}: \mu_{1}(\varphi, \eta) & =\left[S^{-1}\left\{s^{\delta} S\left(H_{0}(\mu)\right)\right\}\right]=\varphi \frac{\left(-\eta^{\delta}\right)}{\Gamma(\delta+1)}, \\
p^{2}: \mu_{2}(\varphi, \eta) & =\left[S^{-1}\left\{s^{\delta} S\left(H_{1}(\mu)\right)\right\}\right]=\varphi \frac{\left(-\eta^{\delta}\right)^{2}}{\Gamma(2 \delta+1)}, \\
p^{3}: \mu_{3}(\varphi, \eta) & =\left[S^{-1}\left\{s^{\delta} S\left(H_{2}(\mu)\right)\right\}\right]=\varphi \frac{\left(-\eta^{\delta}\right)^{3}}{\Gamma(3 \delta+1)}, \\
& : \\
p^{n}: \mu_{n}(\varphi, \eta) & =\left[S^{-1}\left\{s^{\delta} S\left(H_{n-1}(\mu)\right)\right\}\right]=\varphi \frac{\left(-\eta^{\delta}\right)^{n}}{\Gamma(n \delta+1)}
\end{aligned}
$$

Then the series form result is defined as

$$
\mu(\varphi, \eta)=\sum_{n=0}^{\infty} p^{n} \mu_{n}(\varphi, \eta)
$$

The approximate solution, we can achieve as

$$
\begin{aligned}
\mu(\varphi, \eta)= & e^{-\varphi}+\varphi\left\{1-\frac{\eta^{\delta}}{\Gamma(\delta+1)}+\frac{\eta^{2 \delta}}{\Gamma(2 \delta+1)}\right. \\
& \left.-\frac{\eta^{3 \delta}}{\Gamma(3 \delta+1)}+\cdots+\frac{\left(-\eta^{\delta}\right)^{n}}{\Gamma(n \delta+1)}\right\} \\
= & \varphi \sum_{k=0}^{\infty} \frac{\left(\eta^{\delta}\right)^{k}}{\Gamma(k \delta+1)} . \\
\mu(\varphi, \eta)= & e^{-\varphi}+\varphi E_{\delta}\left(\eta^{\delta}\right) .
\end{aligned}
$$

The actual solution is

$$
\mu(\varphi, \eta)=e^{-\varphi}+\varphi e^{-\eta}
$$

Figure 1 shows the exact and the analytical solution figures at $\delta=1$ of Example 1. Figure 1 shows that both the analytical and actual solutions are in close contact with each other. Figure 2 shows that the first graph analytical solution of fractional-order at $\delta=0.8$, and the second graph fractional order $\delta=0.6$. In Figure 3, the first graph shows the approximate solution of fractional-order at $\delta=0.4$, and the second graph shows the different fractional-order $\delta$ of Example 1. In Figure 4, the 2D graphs show that the first actual and approximate solution and second graph show the different fractional-order $\delta$ of Example 1. The solution is very rapidly convergent by using the homotopy perturbation method and the iterative method by modifying the Shehu transformation. It may be concluded that both methodologies are efficient and very powerful to find the analytical result as well as approximate results of various physical fractional problems.

4.2. Example. Consider the fractional Cauchy-reaction diffusion equation is given as:

$$
D_{\eta}^{\delta} \mu(\varphi, \eta)=D_{\eta}^{2} \mu(\varphi, \eta)-\left(1+4 \varphi^{2}\right) \mu(\varphi, \eta), 0<\delta \leq 1,
$$

with initial condition

$$
\mu(\varphi, 0)=e^{\varphi^{2}},
$$

and the exact solution is given as

$$
\mu(\varphi, \eta)=e^{\varphi^{2}+1} .
$$

First, we implement the NITM, we obtain as

$$
\begin{aligned}
\mu_{0}(\varphi, \eta) & =e^{\varphi^{2}} \\
\mu_{1}(\varphi, \eta) & =S^{-1}\left[s^{\delta} S\left\{D_{\eta}^{2} \mu_{0}(\varphi, \eta)-\left(1+4 \varphi^{2}\right) \mu_{0}(\varphi, \eta)\right\}\right] \\
& =e^{\varphi^{2}} \frac{\eta^{\delta}}{\Gamma(\delta+1)}
\end{aligned}
$$



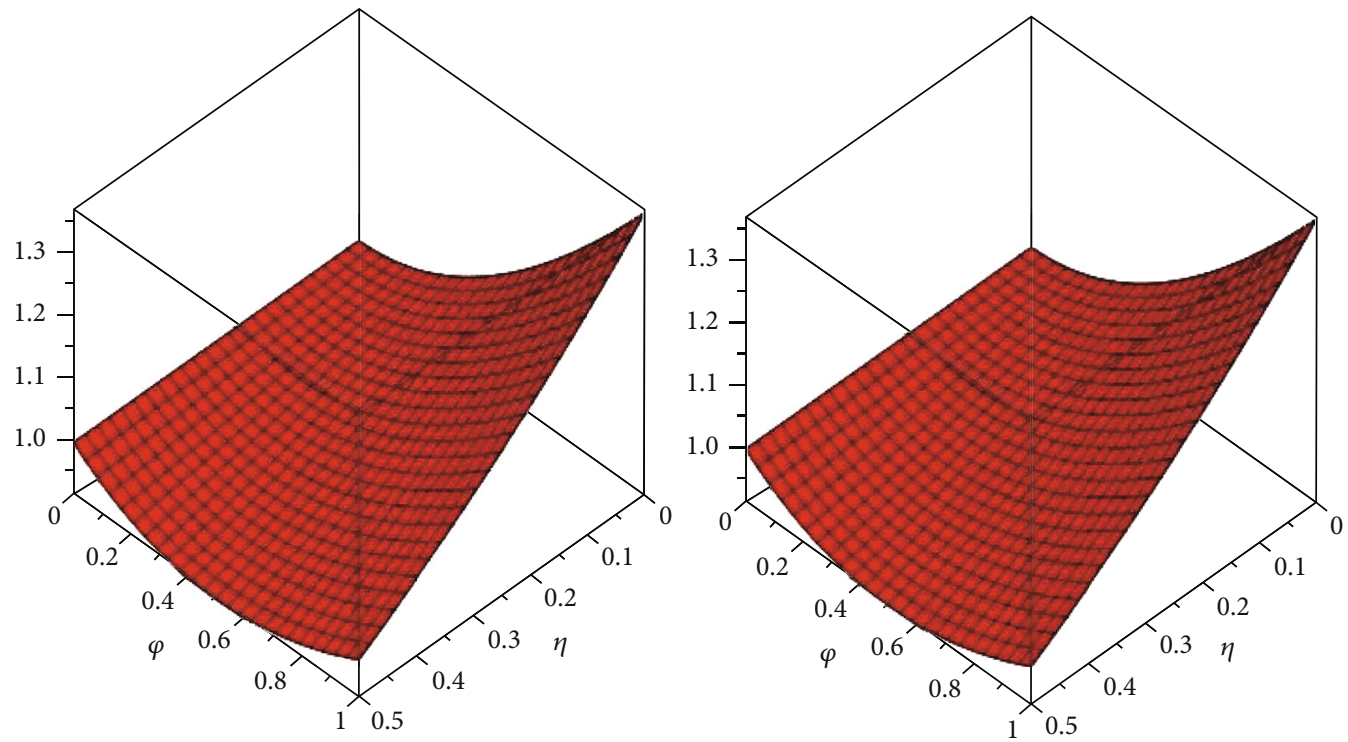

FIgURE 1: The first graph shows the exact and second analytical solution figures at $\delta=1$ of Example 1.
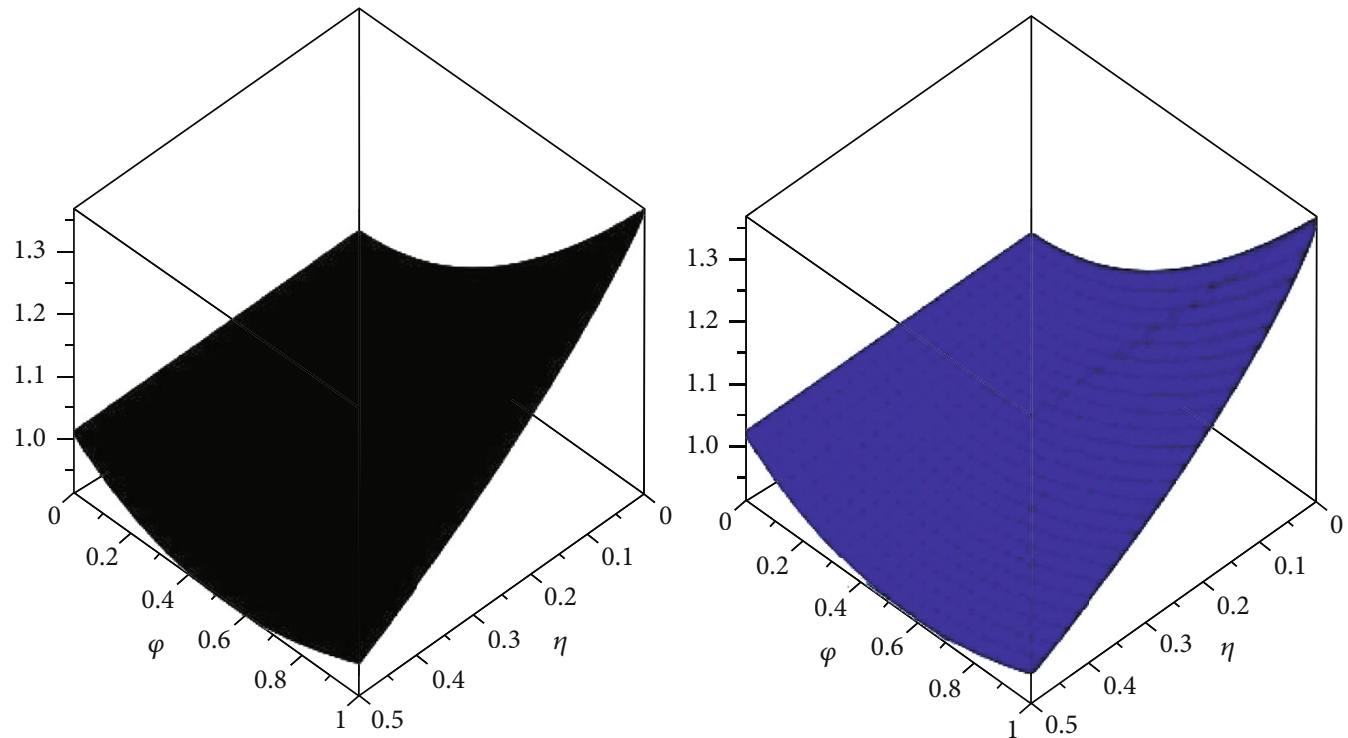

Figure 2: The first graph shows the analytical solution of fractional-order at $\delta=0.8$ and second graph fractional order 0.6 of Example 1 .

$$
\begin{aligned}
\mu_{2}(\varphi, \eta) & =S^{-1}\left[s^{\delta} S\left\{D_{\eta}^{2} \mu_{1}(\varphi, \eta)-\left(1+4 \varphi^{2}\right) \mu_{1}(\varphi, \eta)\right\}\right] \\
& =e^{\varphi^{2}} \frac{\eta^{2 \delta}}{\Gamma(2 \delta+1)}, \\
\mu_{3}(\varphi, \eta) & =S^{-1}\left[s^{\delta} S\left\{D_{\eta}^{2} \mu_{2}(\varphi, \eta)-\left(1+4 \varphi^{2}\right) \mu_{2}(\varphi, \eta)\right\}\right] \\
& =e^{\varphi^{2}} \frac{\eta^{3 \delta}}{\Gamma(3 \delta+1)},
\end{aligned}
$$

$$
\begin{aligned}
\mu_{n}(\varphi, \eta) & =S^{-1}\left[s^{\delta} S\left\{D_{\eta}^{2} \mu_{n}(\varphi, \eta)-\left(1+4 \varphi^{2}\right) \mu_{n}(\varphi, \eta)\right\}\right] \\
& =e^{\varphi^{2}} \frac{\left(\eta^{\delta}\right)^{n}}{\Gamma(n \delta+1)}, n \geq 0 .
\end{aligned}
$$

The series form of iterative transform method is defined as

$$
\begin{aligned}
\mu(\varphi, \eta)= & \mu_{0}(\varphi, \eta)+\mu_{1}(\varphi, \eta)+\mu_{2}(\varphi, \eta) \\
& +\mu_{3}(\varphi, \eta)+\cdots \mu_{n}(\varphi, \eta) .
\end{aligned}
$$



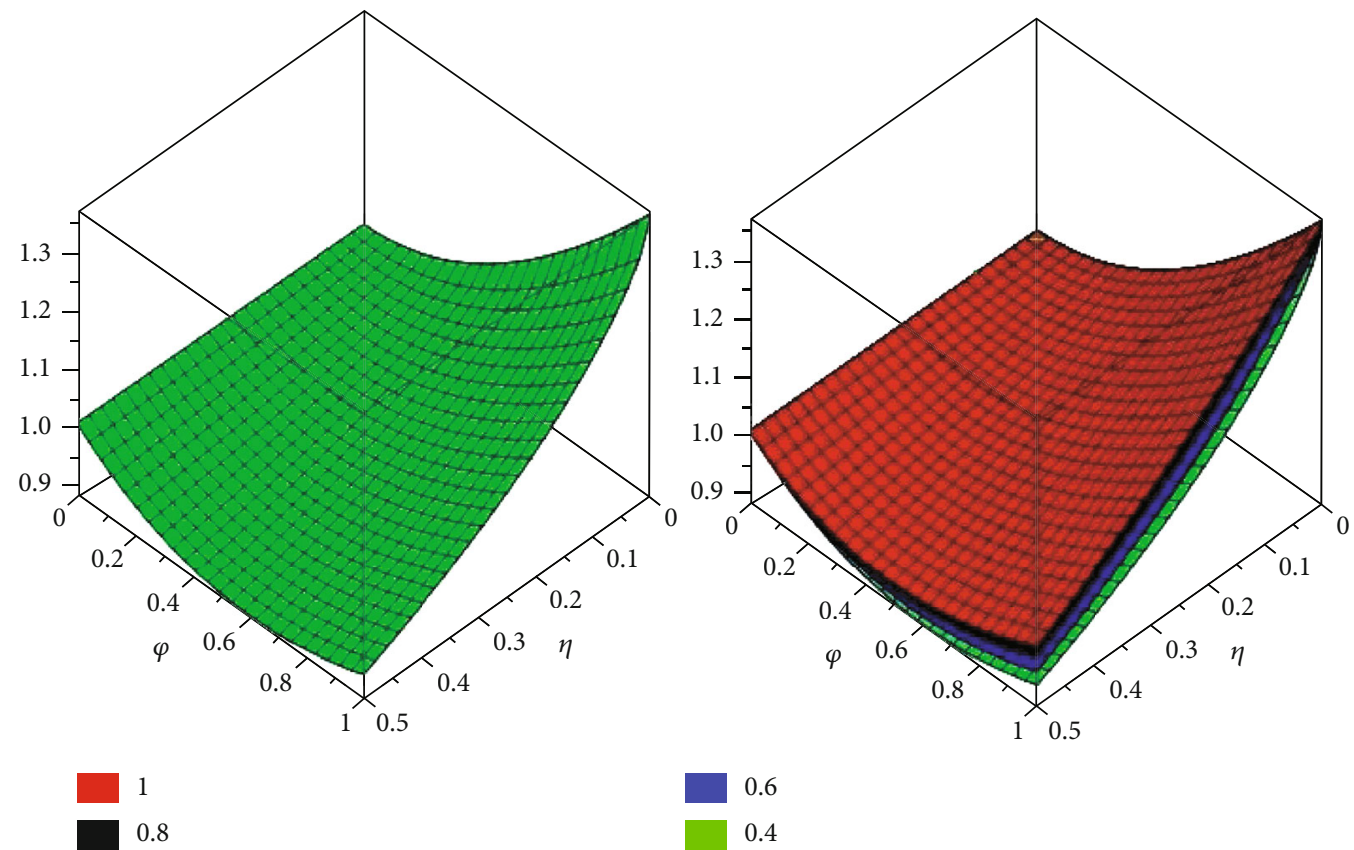

Figure 3: The first graph shows the analytical solution of fractional-order at $\delta=0.4$, and the second graph shows the different fractional-order $\delta$ of Example 1.
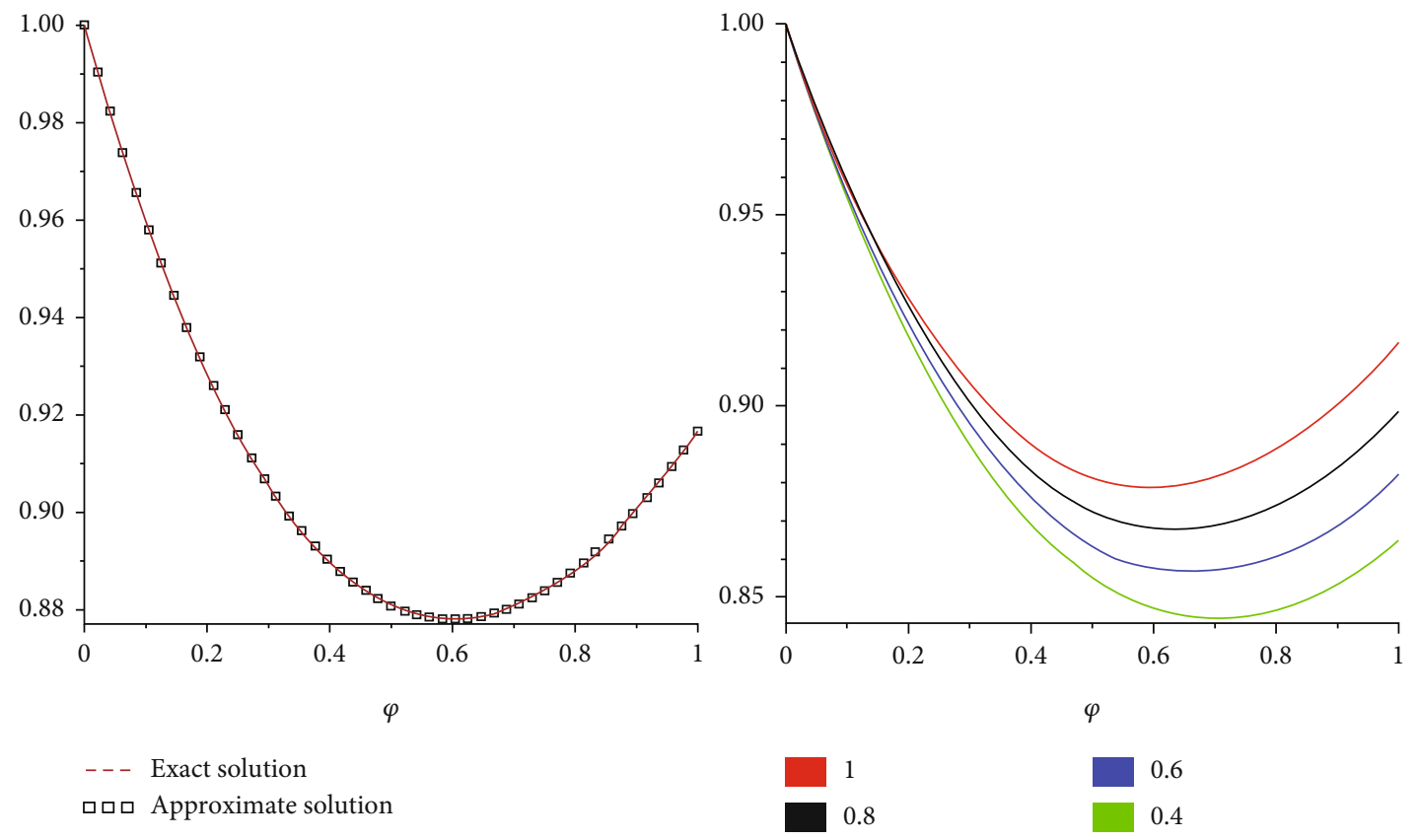

FIgURE 4: The first graph shows the exact and analytical solution, and the second graph shows the different fractional-order $\delta$ of Example 1 .

The approximate solution is given as

$$
\begin{aligned}
\mu(\varphi, \eta)= & e^{\varphi^{2}}\left\{1+\frac{\eta^{\delta}}{\Gamma(\delta+1)}+\frac{\eta^{2 \delta}}{\Gamma(2 \delta+1)}\right. \\
& \left.+\frac{\eta^{3 \delta}}{\Gamma(3 \delta+1)}+\cdots+\frac{\eta^{\eta \delta}}{\Gamma(n \delta+1)}\right\} . \\
\mu(\varphi, \eta)= & e^{\varphi^{2}} E_{\delta}\left(\eta^{\delta}\right) .
\end{aligned}
$$

Now, we implement HPTM, we get

$$
\sum_{n=0}^{\infty} p^{n} \mu_{n}(\varphi, \eta)=e^{\varphi^{2}}+p\left\{S^{-1}\left(s^{\delta} S\left[\sum_{n=0}^{\infty} p^{n} H_{n}(\mu)\right]\right)\right\} .
$$

On both sides $p$ Comparisons coefficient, we have

$$
p^{0}: \mu_{0}(\varphi, \eta)=e^{\varphi^{2}}
$$



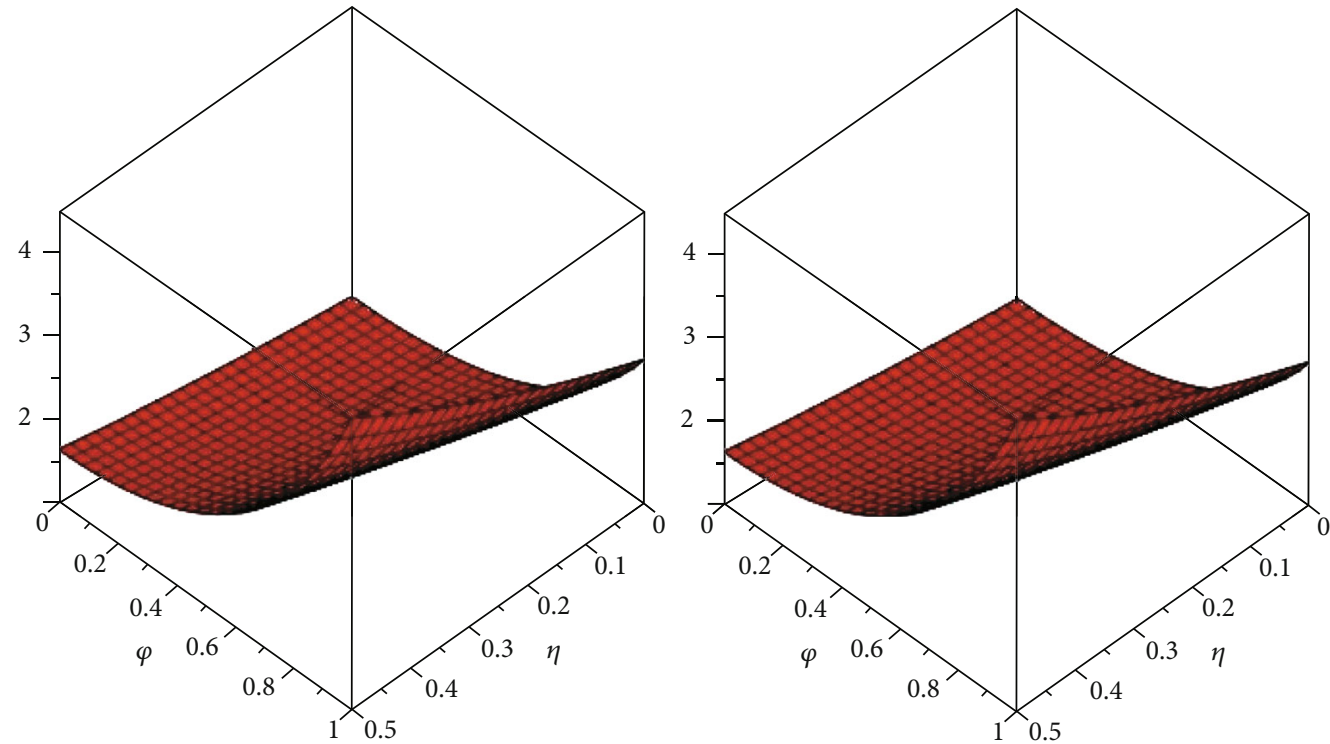

FIgURE 5: The first graph shows the exact and second analytical solution figures at $\delta=1$ of Example 2.

$$
\begin{aligned}
& p^{1}: \mu_{1}(\varphi, \eta)=\left\{S^{-1}\left(s^{\delta} S\left[H_{0}(\mu)\right]\right)\right\}=e^{\varphi^{2}} \frac{\eta^{\delta}}{\Gamma(\delta+1)}, \\
& p^{2}: \mu_{2}(\varphi, \eta)=\left\{S^{-1}\left(s^{\delta} S\left[H_{1}(\mu)\right]\right)\right\}=e^{\varphi^{2}} \frac{\eta^{2 \delta}}{\Gamma(2 \delta+1)}, \\
& p^{3}: \mu_{3}(\varphi, \eta)=\left\{S^{-1}\left(s^{\delta} S\left[H_{2}(\mu)\right]\right)\right\}=e^{\varphi^{2}} \frac{\eta^{3 \delta}}{\Gamma(3 \delta+1)},
\end{aligned}
$$$$
p^{n}: \mu_{n}(\varphi, \eta)=\left\{S^{-1}\left(s^{\delta} S\left[H_{n-1}(\mu)\right]\right)\right\}=e^{\varphi^{2}} \frac{\eta^{n \delta}}{\Gamma(n \delta+1)}
$$

The series form solution is express as

$$
\begin{aligned}
\mu(\varphi, \eta)= & \sum_{n=0}^{\infty} p^{n} \mu_{n}(\varphi, \eta) . \\
\mu(\varphi, \eta)= & e^{\varphi^{2}}\left\{1+\frac{\eta^{\delta}}{\Gamma(\delta+1)}+\frac{\eta^{2 \delta}}{\Gamma(2 \delta+1)}\right. \\
& \left.+\frac{\eta^{3 \delta}}{\Gamma(3 \delta+1)}+\cdots+\frac{\eta^{n \delta}}{\Gamma(n \delta+1)}\right\}, \\
\mu(\varphi, \eta)= & e^{\varphi^{2}} E_{\delta}\left(\eta^{\delta}\right) .
\end{aligned}
$$

Now, $\delta=1$, then the exact result of equation (52) is $\mu(\varphi, \eta)=e^{\varphi^{2}+\eta}$.

Figure 5 shows the exact and the analytical solution figures at $\delta=1$ of Example 2. Figure 5 shows that both the analytical and actual solutions are in close contact with each other. Figure 6 shows the first graph analytical solution of fractional-order at $\delta=0.8$ and the second graph fractional order $\delta=0.6$. In Figure 7, the first graph shows the approxi- mate solution of fractional-order at $\delta=0.4$, and the second graph shows the different fractional-order $\delta$ of Example 2. In Figure 8, the 2D graphs show that the first actual and approximate solution and second graph show the different fractional-order $\delta$ of Example 2. The solution is very rapidly convergent by using the homotopy perturbation method and the iterative method by modifying the Shehu transformation. It may be concluded that both methodologies are efficient and very powerful to find the analytical solution as well as approximate results of various physical fractional problems.

4.3. Example. Consider fractional-order Cauchy-reaction diffusion equation:

$$
D_{\eta}^{\delta} \mu(\varphi, \eta)=D_{\eta}^{2} \mu(\varphi, \eta)+2 \eta \mu(\varphi, \eta), 0<\delta \leq 1
$$

with initial condition

$$
\mu(\varphi, 0)=e^{\varphi}
$$

the actual solution is

$$
\mu(\varphi, \eta)=e^{\varphi+\eta+\eta^{2}}
$$

Apply the Shehu transform, we obtain as

$$
\mu(\varphi, \eta)=e^{\varphi}+S^{-1}\left[s^{\delta} S\left(D_{\eta}^{2} \mu(\varphi, \eta)+2 \eta \mu(\varphi, \eta)\right)\right] .
$$

First, we implement the NITM

$$
\mu_{0}(\varphi, \eta)=e^{\varphi}
$$



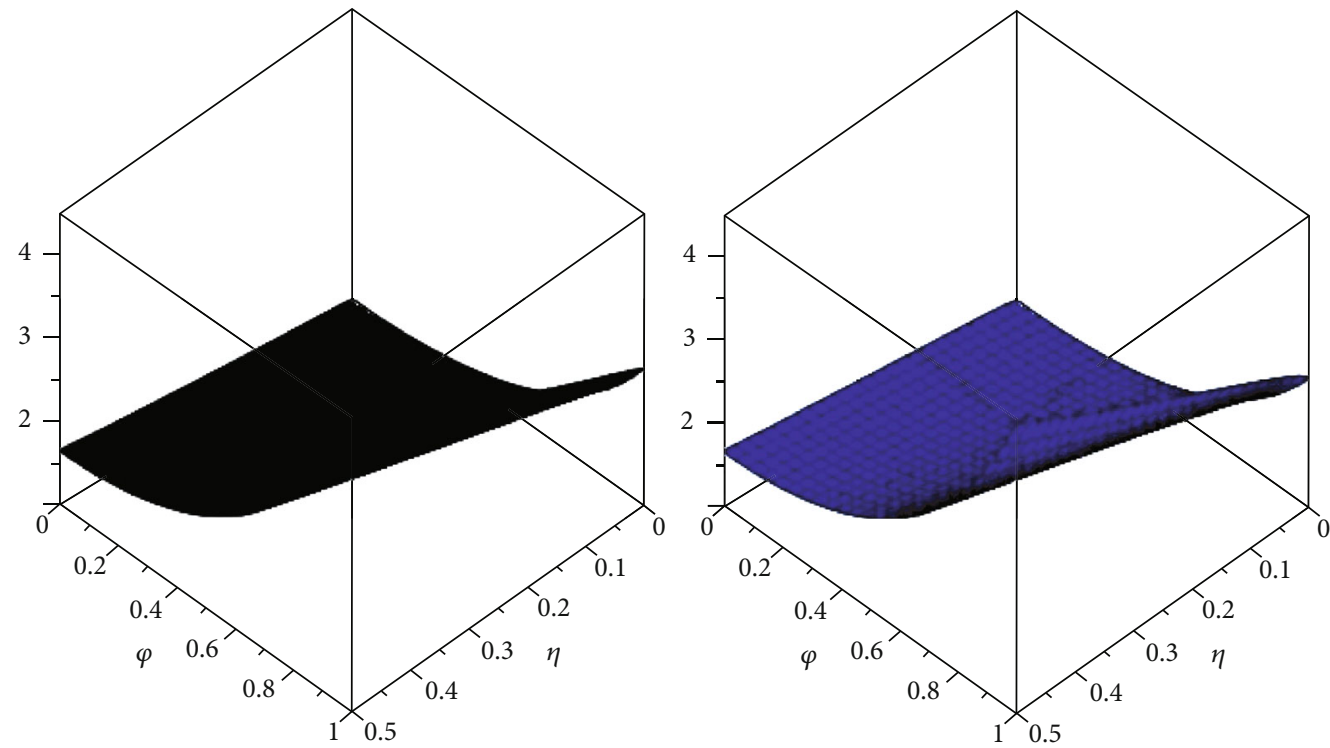

FIGURE 6: The first graph shows the analytical solution of fractional-order at $\delta=0.8$ and second graph fractional order 0.6 of Example 2.
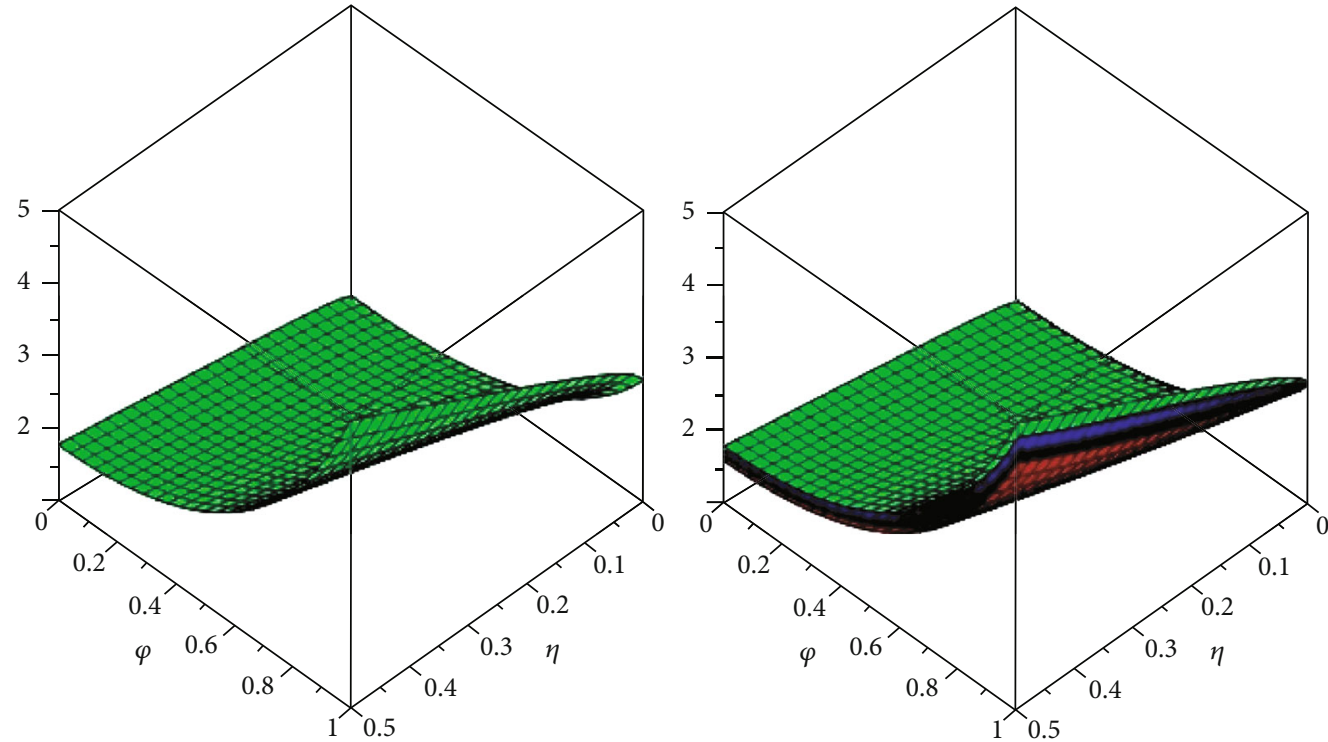

1
0.8

FIGURE 7: The first graph shows the analytical solution of fractional-order at $\delta=0.4$, and the second graph shows the different fractional-order $\delta$ of Example 2.

$$
\begin{aligned}
\mu_{1}(\varphi, \eta) & =S^{-1}\left[s^{\delta} S\left\{D_{\eta}^{2} \mu_{0}(\varphi, \eta)+2 \eta \mu_{0}(\varphi, \eta)\right\}\right] \\
& =e^{\varphi}\left(\frac{\eta^{\delta}}{\Gamma(\delta+1)}+\frac{2 \eta^{\delta+1}}{\Gamma(\delta+2)}\right), \\
\mu_{2}(\varphi, \eta)=S^{-1}\left[s^{\delta} S\left\{D_{\eta}^{2} \mu_{1}(\varphi, \eta)+2 \eta \mu_{1}(\varphi, \eta)\right\}\right] & \\
= & e^{\varphi}\left(\frac{\eta^{2 \delta}}{\Gamma(2 \delta+1)}+\frac{2(\delta+2) \eta^{2 \delta+1}}{\Gamma(2 \delta+2)}+\frac{4(\delta+2) \eta^{2 \delta+2}}{\Gamma(2 \delta+3)}\right),
\end{aligned}
$$

$$
\begin{aligned}
\mu_{3}(\varphi, \eta)= & S^{-1}\left[s^{\delta} S\left\{D_{\eta}^{2} \mu_{2}(\varphi, \eta)+2 \eta \mu_{2}(\varphi, \eta)\right\}\right] \\
= & e^{\varphi}\left(\frac{\eta^{3 \delta}}{\Gamma(3 \delta+1)}+\frac{6(\delta+1) \eta^{3 \delta+1}}{\Gamma(3 \delta+2)}\right. \\
& \left.+\frac{4(\delta+2)(\delta+3) \eta^{3 \delta+2}}{\Gamma(3 \delta+3)}+\frac{8(\delta+2)(2 \delta+3) \eta^{3 \delta+3}}{\Gamma(3 \delta+4)}\right)
\end{aligned}
$$




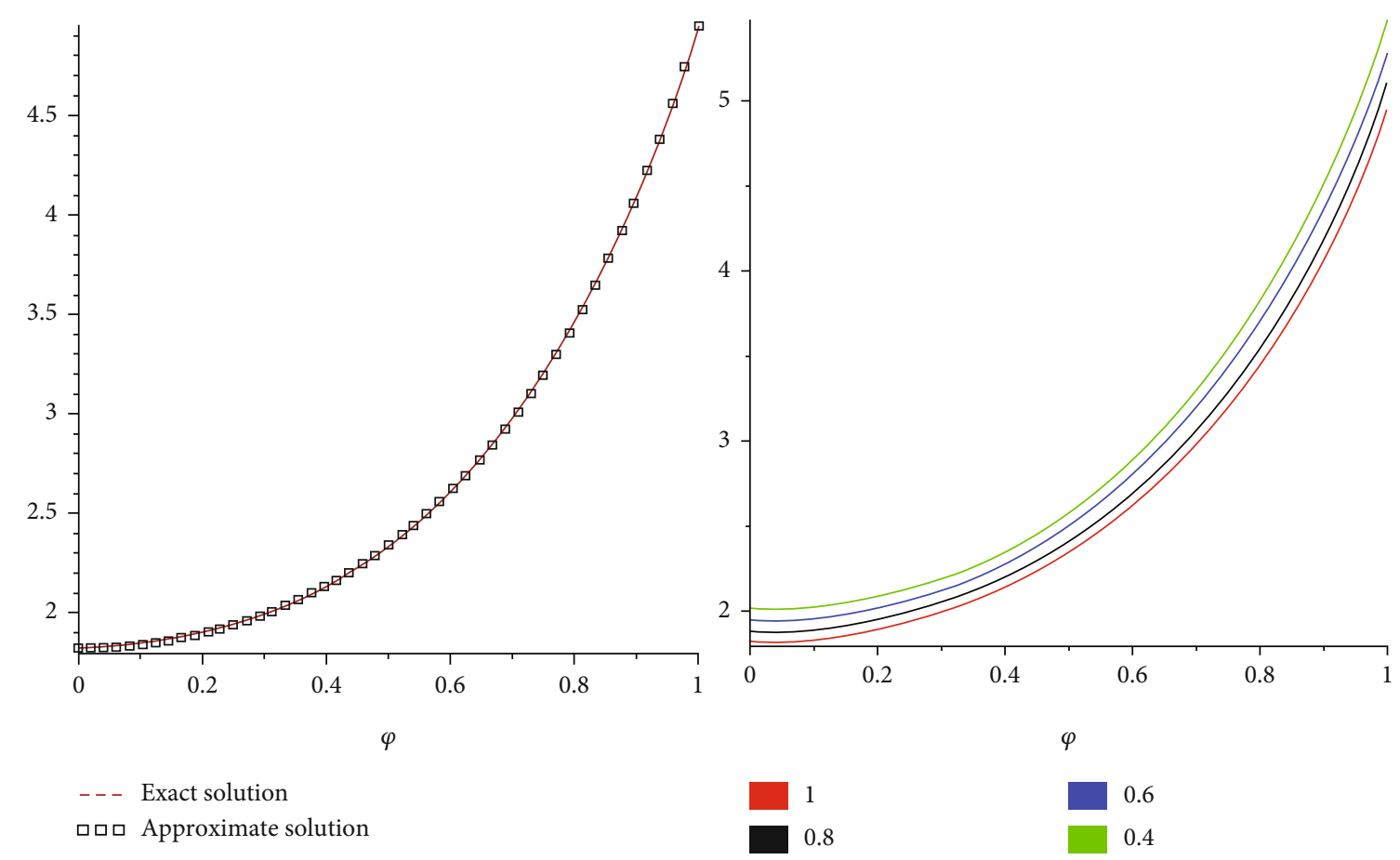

FIgURE 8: The first graph shows the exact and analytical solution, and the second graph shows the different fractional-order $\delta$ of Example 2.

The series form of proposed technique can be defined as

$\mu(\varphi, \eta)=\mu_{0}(\varphi, \eta)+\mu_{1}(\varphi, \eta)+\mu_{2}(\varphi, \eta)+\mu_{3}(\varphi, \eta)+\cdots \mu_{n}(\varphi, \eta)$

The approximate solution is given as

$$
\begin{aligned}
\mu(\varphi, \eta)= & e^{\varphi}+e^{\varphi}\left(\frac{\eta^{\delta}}{\Gamma(\delta+1)}+\frac{2 \eta^{\delta+1}}{\Gamma(\delta+2)}\right) \\
& +e^{\varphi}\left(\frac{\eta^{2 \delta}}{\Gamma(2 \delta+1)}+\frac{2(\delta+2) \eta^{2 \delta+1}}{\Gamma(2 \delta+2)}+\frac{4(\delta+2) \eta^{2 \delta+2}}{\Gamma(2 \delta+3)}\right) \\
& +e^{\varphi}\left(\frac{\eta^{3 \delta}}{\Gamma(3 \delta+1)}+\frac{6(\delta+1) \eta^{3 \delta+1}}{\Gamma(3 \delta+2)}+\frac{4(\delta+2)(\delta+3) \eta^{3 \delta+2}}{\Gamma(3 \delta+3)}\right. \\
& \left.+\frac{8(\delta+2)(2 \delta+3) \eta^{3 \delta+3}}{\Gamma(3 \delta+4)}\right)+\cdots
\end{aligned}
$$

Now, we apply HPTM, we get

$$
\sum_{n=0}^{\infty} p^{n} \mu_{n}(\varphi, \eta)=e^{\varphi}+p\left[S^{-1}\left\{s^{\delta} S\left(\sum_{n=0}^{\infty} p^{n} H_{n}(\mu)\right)\right\}\right] .0
$$

The coefficients of $p$ Comparison on both sides, we achieve as

$p^{0}: \mu_{0}(\varphi, \eta)=e^{\varphi}$

$p^{1}: \mu_{1}(\varphi, \eta)=\left[S^{-1}\left\{s^{\delta} S\left(H_{0}(\mu)\right)\right\}\right]=e^{\varphi}\left(\frac{\eta^{\delta}}{\Gamma(\delta+1)}+\frac{2 \eta^{\delta+1}}{\Gamma(\delta+2)}\right)$,

$$
\begin{aligned}
p^{2} & : \mu_{2}(\varphi, \eta) \\
= & {\left[S^{-1}\left\{s^{\delta} S\left(H_{1}(\mu)\right)\right\}\right] } \\
& =e^{\varphi}\left(\frac{\eta^{2 \delta}}{\Gamma(2 \delta+1)}+\frac{2(\delta+2) \eta^{2 \delta+1}}{\Gamma(2 \delta+2)}+\frac{4(\delta+2) \eta^{2 \delta+2}}{\Gamma(2 \delta+3)}\right), \\
p^{3}: & \mu_{3}(\varphi, \eta) \\
= & {\left[S^{-1}\left\{s^{\delta} S\left(H_{2}(\mu)\right)\right\}\right] } \\
= & e^{\varphi}\left(\frac{\eta^{3 \delta}}{\Gamma(3 \delta+1)}+\frac{6(\delta+1) \eta^{3 \delta+1}}{\Gamma(3 \delta+2)}+\frac{4(\delta+2)(\delta+3) \eta^{3 \delta+2}}{\Gamma(3 \delta+3)}\right. \\
& \left.+\frac{8(\delta+2)(2 \delta+3) \eta^{3 \delta+3}}{\Gamma(3 \delta+4)}\right) .
\end{aligned}
$$

Finally, we obtain the analytical result $\mu(\varphi, \eta)$ to the truncate series.

$$
\begin{aligned}
\mu(\varphi, \eta)= & \lim _{N \longrightarrow \infty} \sum_{n=1}^{N} \mu_{n}(\varphi, \eta) . \\
\mu(\varphi, \eta)= & e^{\varphi}+e^{\varphi}\left(\frac{\eta^{\delta}}{\Gamma(\delta+1)}+\frac{2 \eta^{\delta+1}}{\Gamma(\delta+2)}\right) \\
& +e^{\varphi}\left(\frac{\eta^{2 \delta}}{\Gamma(2 \delta+1)}+\frac{2(\delta+2) \eta^{2 \delta+1}}{\Gamma(2 \delta+2)}+\frac{4(\delta+2) \eta^{2 \delta+2}}{\Gamma(2 \delta+3)}\right) \\
& +e^{\varphi}\left(\frac{\eta^{3 \delta}}{\Gamma(3 \delta+1)}+\frac{6(\delta+1) \eta^{3 \delta+1}}{\Gamma(3 \delta+2)}\right. \\
& \left.+\frac{4(\delta+2)(\delta+3) \eta^{3 \delta+2}}{\Gamma(3 \delta+3)}+\frac{8(\delta+2)(2 \delta+3) \eta^{3 \delta+3}}{\Gamma(3 \delta+4)}\right)+\cdots
\end{aligned}
$$



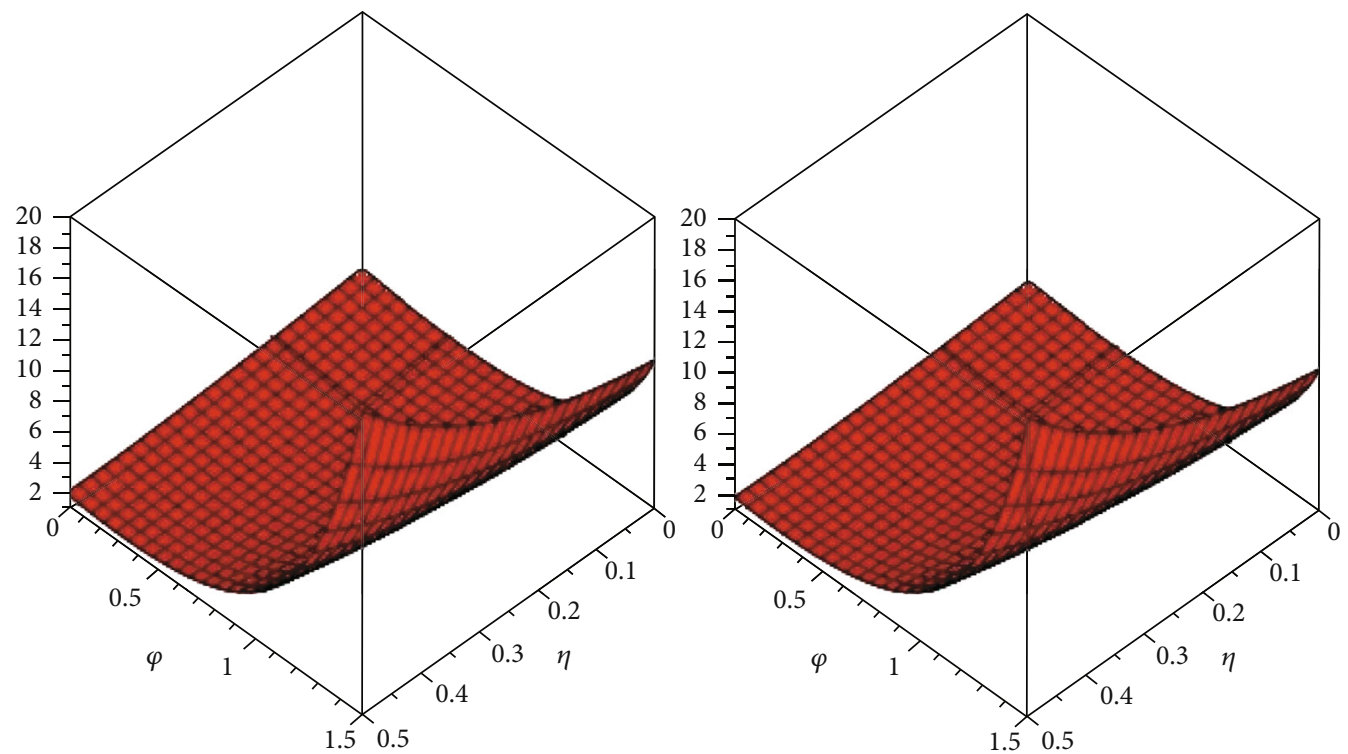

FIGURE 9: The first graph shows the exact and second analytical solution figures at $\delta=1$ of Example 3.
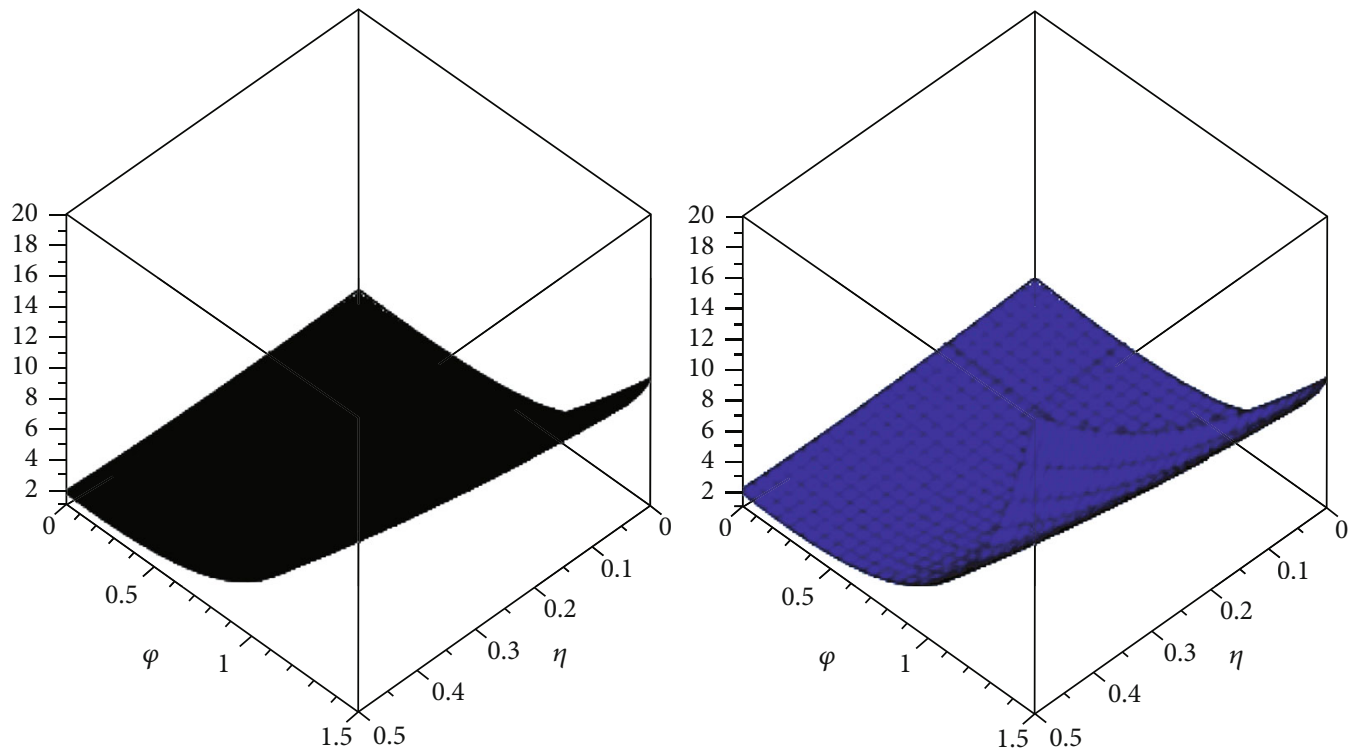

FIGURE 10: The first graph shows the analytical solution of fractional-order at $\delta=0.8$ and second graph fractional order 0.6 of Example 3 .

Now, for $\delta=1$, the actual result of the above series is

$$
\mu(\varphi, \eta)=e^{\varphi+\eta+\eta^{2}}
$$

Figure 9 shows the exact and the analytical solution figures at $\delta=1$ of Example 3. Figure 9 shows that both the analytical and actual solutions are in close contact with each other. Figure 10 shows that the first graph analytical solution of fractional-order at $\delta=0.8$ and second graph fractional order $\delta=0.6$. In Figure 11, the first graph shows the approximate solution of fractional-order at $\delta=0.4$, and the second graph shows the different fractional-order $\delta$ of Example 3. The solution is very rapidly convergent by using the Homotopy perturbation method and the iterative method by mod- ifying the Shehu transformation. It may be concluded that both methodologies are efficient and very powerful to find the analytical result as well as approximate results of various physical fractional problems.

4.4. Example. Consider the fractional Cauchy-reaction diffusion equation is given as:

$$
D_{\eta}^{\delta} \mu(\varphi, \eta)=D_{\eta}^{2} \mu(\varphi, \eta)-\left(4 \varphi^{2}-2 \eta+2\right) \mu(\varphi, \eta), 0<\delta \leq 1
$$

with initial condition

$$
\mu(\varphi, 0)=e^{\varphi^{2}}
$$



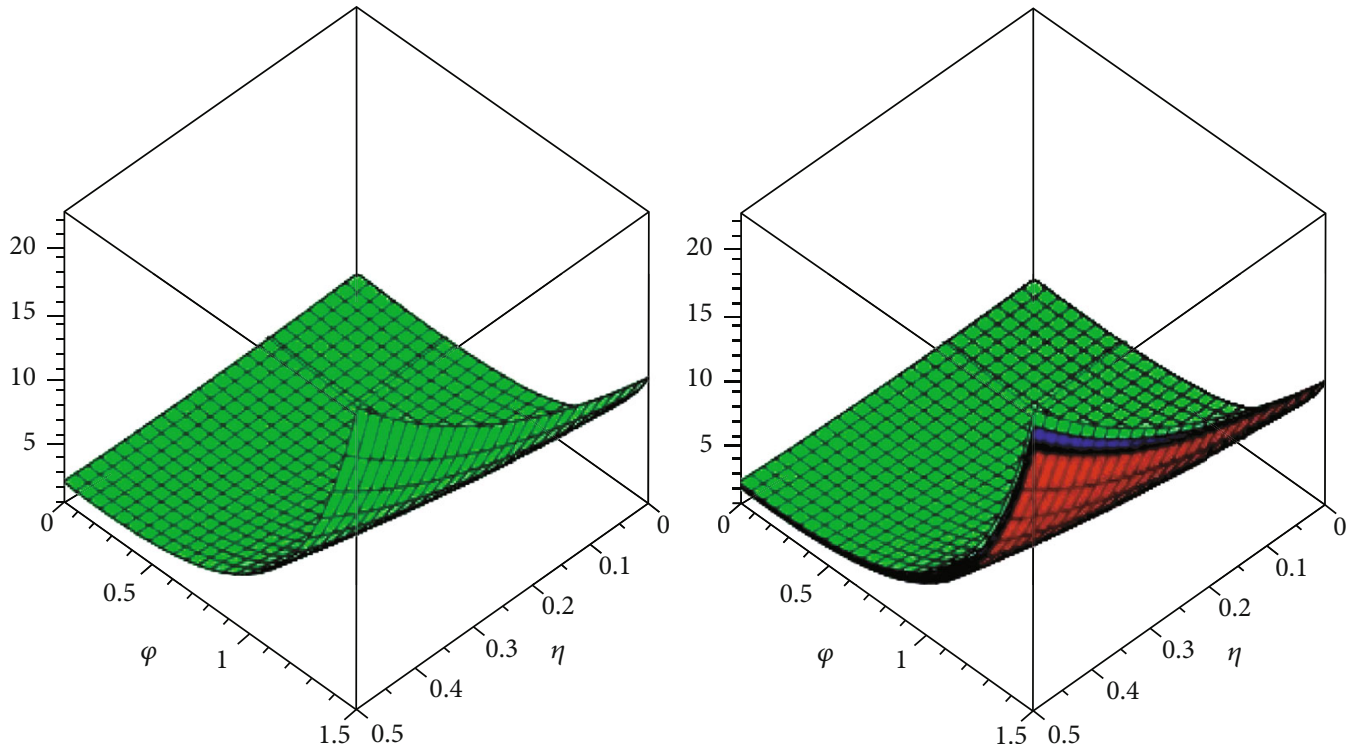

1
0.8

0.6

0.4

FIGURE 11: The first graph shows the analytical solution of fractional-order at $\delta=0.4$, and the second graph shows the different fractionalorder $\delta$ of Example 3.

the exact result is

$$
\mu(\varphi, \eta)=e^{\varphi^{2}+\eta^{2}}
$$

First, we apply the NITM

$$
\mu_{0}(\varphi, \eta)=e^{\varphi^{2}}
$$

$$
\begin{aligned}
\mu_{1}(\varphi, \eta) & =S^{-1}\left[s^{\delta} S\left\{D_{\eta}^{2} \mu_{0}(\varphi, \eta)-\left(4 \varphi^{2}-2 \eta+2\right) \mu_{0}(\varphi, \eta)\right\}\right] \\
& =2 e^{\varphi^{2}} \frac{\eta^{\delta+1}}{\Gamma(\delta+2)}
\end{aligned}
$$

$$
\begin{aligned}
\mu_{2}(\varphi, \eta) & =S^{-1}\left[s^{\delta} S\left\{D_{\eta}^{2} \mu_{1}(\varphi, \eta)-\left(4 \varphi^{2}-2 \eta+2\right) \mu_{1}(\varphi, \eta)\right\}\right] \\
& =2^{2} e^{\varphi^{2}} \frac{(\delta+2) \eta^{2(\delta+1)}}{\Gamma(2 \delta+3)}
\end{aligned}
$$

$$
\begin{aligned}
\mu_{3}(\varphi, \eta) & =S^{-1}\left[s^{\delta} S\left\{D_{\eta}^{2} \mu_{2}(\varphi, \eta)-\left(4 \varphi^{2}-2 \eta+2\right) \mu_{2}(\varphi, \eta)\right\}\right] \\
& =2^{3} e^{\varphi^{2}} \frac{(\delta+2)(2 \delta+3) \eta^{3(\delta+1)}}{\Gamma(3 \delta+4)}
\end{aligned}
$$

The series form result is defined as

$\mu(\varphi, \eta)=\mu_{0}(\varphi, \eta)+\mu_{1}(\varphi, \eta)+\mu_{2}(\varphi, \eta)+\mu_{3}(\varphi, \eta)+\cdots \mu_{n}(\varphi, \eta)$.
The approximate solution is

$$
\begin{aligned}
\mu(\varphi, \eta)= & e^{\varphi^{2}}+2 e^{\varphi^{2}} \frac{\eta^{\delta+1}}{\Gamma(\delta+2)}+2^{2} e^{\varphi^{2}} \frac{(\delta+2) \eta^{2(\delta+1)}}{\Gamma(2 \delta+3)} \\
& +2^{3} e^{\varphi^{2}} \frac{(\delta+2)(2 \delta+3) \eta^{3(\delta+1)}}{\Gamma(3 \delta+4)} \\
& +2^{4} e^{\varphi^{2}} \frac{(\delta+2)(2 \delta+3)(3 \delta+4) \eta^{4(\delta+1)}}{\Gamma(4 \delta+5)}+\cdots
\end{aligned}
$$

Now, implement the HPTM, we obtain as

$$
\sum_{n=0}^{\infty} p^{n} \mu_{n}(\varphi, \eta)=e^{\varphi^{2}}+p\left[S^{-1}\left\{s^{\delta} S\left(\sum_{n=0}^{\infty} p^{n} H_{n}(\mu)\right)\right\}\right]
$$

The comparison of coefficient of $p$ on both sides, we get $p^{0}: \mu_{0}(\varphi, \eta)=e^{\varphi^{2}}$

$$
\begin{aligned}
& p^{1}: \mu_{1}(\varphi, \eta)=\left[S^{-1}\left\{s^{\delta} S\left(H_{0}(\mu)\right)\right\}\right]=2 e^{\varphi^{2}} \frac{\eta^{\delta+1}}{\Gamma(\delta+2)} \\
& p^{2}: \mu_{2}(\varphi, \eta)=\left[S^{-1}\left\{s^{\delta} S\left(H_{1}(\mu)\right)\right\}\right]=2^{2} e^{\varphi^{2}} \frac{(\delta+2) \eta^{2(\delta+1)}}{\Gamma(2 \delta+3)},
\end{aligned}
$$

$$
\begin{aligned}
p^{3}: \mu_{3}(\varphi, \eta) & =\left[S^{-1}\left\{s^{\delta} S\left(H_{2}(\mu)\right)\right\}\right] \\
& =2^{3} e^{\varphi^{2}} \frac{(\delta+2)(2 \delta+3) \eta^{3(\delta+1)}}{\Gamma(3 \delta+4)},
\end{aligned}
$$



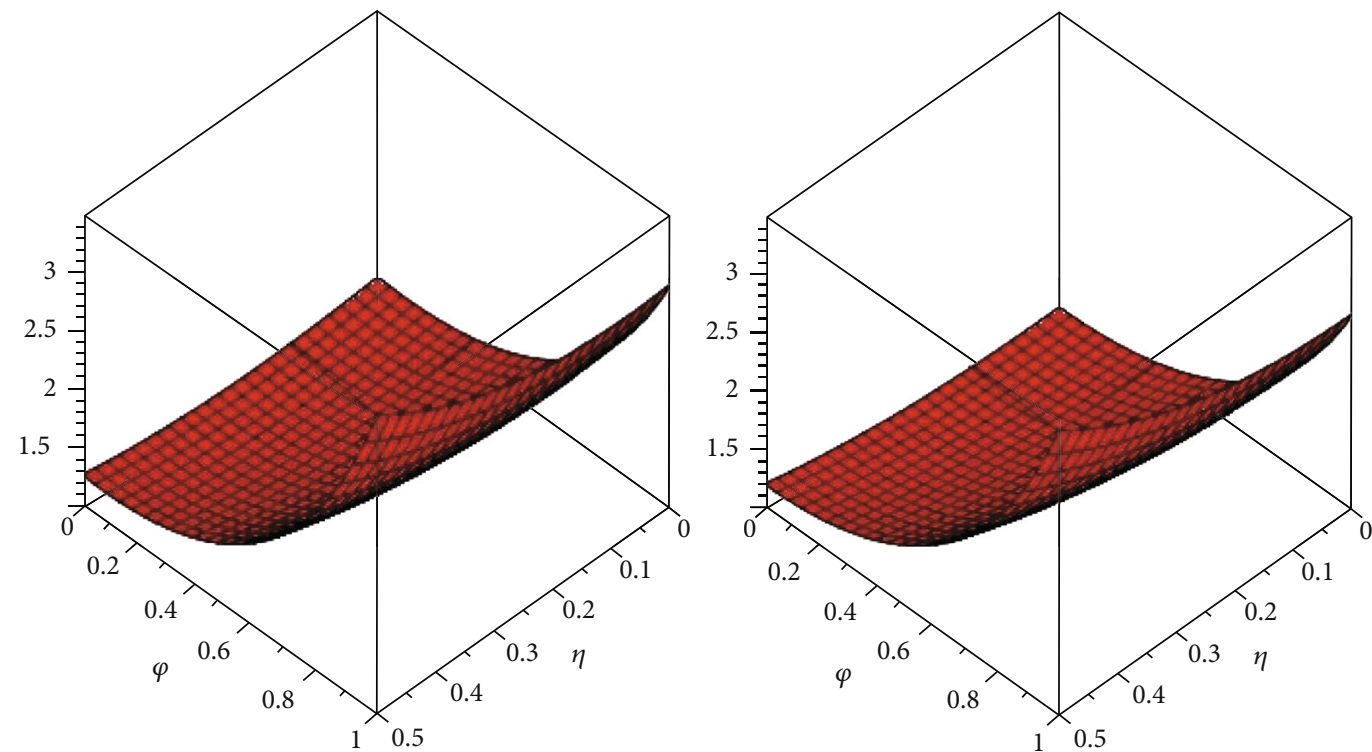

Figure 12: The first graph shows the exact and second analytical solution figures at $\delta=1$ of Example 4 .
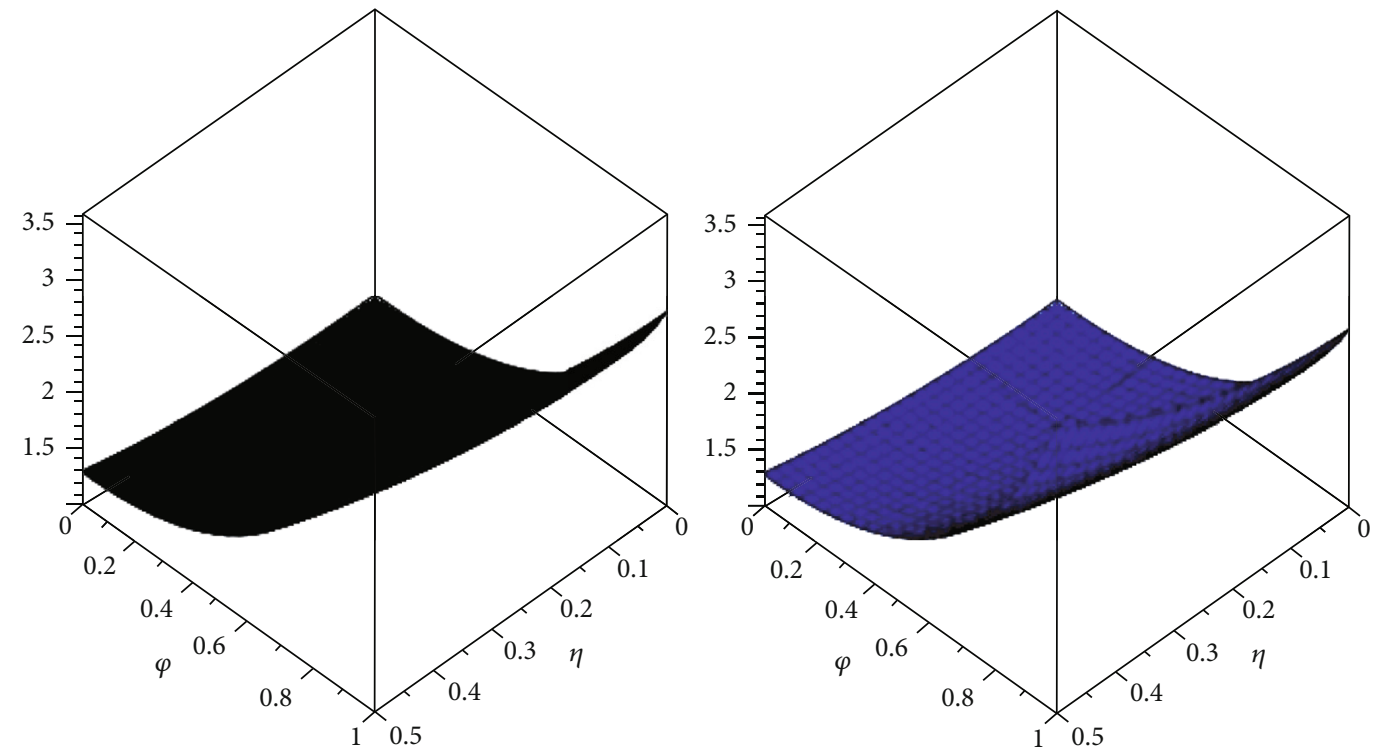

FIGURE 13: The first graph shows the analytical solution of fractional-order at $\delta=0.8$ and second graph fractional order 0.6 of Example 4 .

$$
\begin{aligned}
p^{4}: \mu_{4}(\varphi, \eta) & =\left[S^{-1}\left\{s^{\delta} S\left(H_{3}(\mu)\right)\right\}\right] \\
& =2^{4} e^{\varphi^{2}} \frac{(\delta+2)(2 \delta+3)(3 \delta+4) \eta^{4(\delta+1)}}{\Gamma(4 \delta+5)} .
\end{aligned}
$$

Finally, we obtain the approximate result $\mu(\varphi, \eta)$ by truncate series

$$
\mu(\varphi, \eta)=\lim _{N \longrightarrow \infty} \sum_{n=1}^{N} \mu_{n}(\varphi, \eta)
$$

$$
\begin{aligned}
\mu(\varphi, \eta)= & e^{\varphi^{2}}+2 e^{\varphi^{2}} \frac{\eta^{\delta+1}}{\Gamma(\delta+2)}+2^{2} e^{\varphi^{\varphi^{2}}} \frac{(\delta+2) \eta^{2(\delta+1)}}{\Gamma(2 \delta+3)} \\
& +2^{3} e^{\varphi^{2}} \frac{(\delta+2)(2 \delta+3) \eta^{3(\delta+1)}}{\Gamma(3 \delta+4)} \\
& +2^{4} e^{\varphi^{2}} \frac{(\delta+2)(2 \delta+3)(3 \delta+4) \eta^{4(\delta+1)}}{\Gamma(4 \delta+5)}+\cdots
\end{aligned}
$$

The special case for $\delta=1$, the close form above equation is expressed as

$$
\mu(\varphi, \eta)=e^{\varphi^{2}+\eta^{2}}
$$



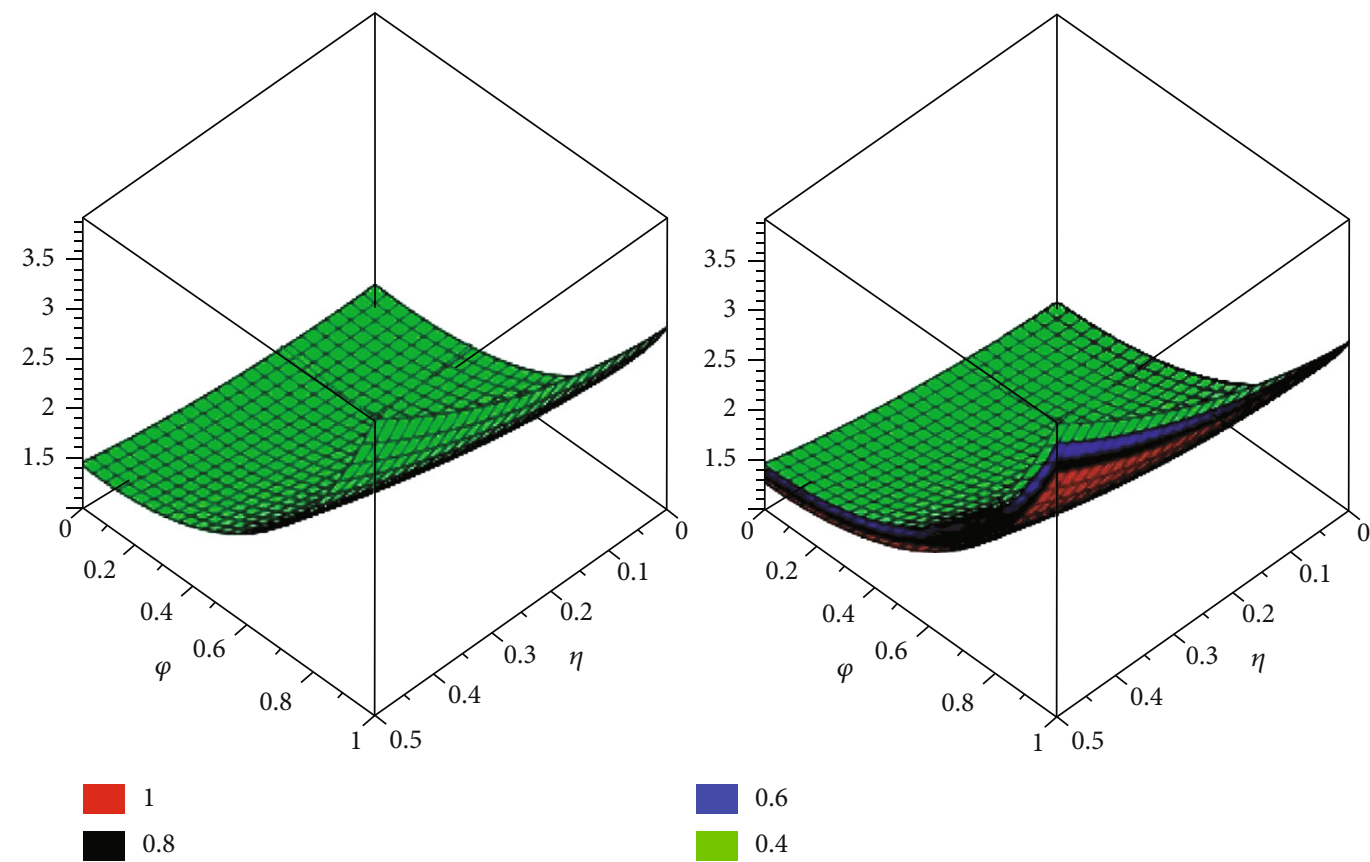

0.6

0.4

FIGURE 14: The first graph shows the analytical solution of fractional-order at $\delta=0.4$, and the second graph shows the different fractionalorder $\delta$ of Example 4 .

Figure 12 shows the exact and the analytical solution figures at $\delta=1$ of Example 4. Figure 12 shows that both the analytical and actual solutions are in close contact with each other. Figure 13 shows the first graph analytical solution of fractional-order at $\delta=0.8$ and second graph fractional order $\delta=0.6$. In Figure 14, the first graph shows the approximate solution of fractional-order at $\delta=0.4$, and the second graph shows the different fractional-order $\delta$ of Example 4. The solution is very rapidly convergent by apply the homotopy perturbation method and the iterative method by modifying the Shehu transformation. It may be concluded that both methodologies are efficient and very powerful in finding the analytical result as well as approximate results of various physical fractional problems.

\section{Conclusion}

This paper develops an effective and new coupling method of the homotopy perturbation method, the iterative method, and Shehu transform approach for the time-fractional Cauchyreaction diffusion equations. With the help of the Shehu transform, the calculation of this method is very straightforward and simple. A very close contact of the achieved solutions with the exact solution of the problem is analyzed. It is also investigated that the rate of convergence of the proposed techniques is accurate the solution of fractional-order partial differential equations. Thus, we further this technique can be applied to solve other schemes of linear and nonlinear fractional-order partial differential equations.

\section{Data Availability}

The data used to support the findings of this study are included within the article.

\section{Conflicts of Interest}

The authors declare that there are no conflicts of interest regarding the publication of this article.

\section{Authors' Contributions}

All authors contributed equally and significantly in writing this article. All authors read and approved the final manuscript.

\section{Acknowledgments}

One of the co-authors (A. M. Zidan) extends their appreciation to the Deanship of Scientific Research at King Khalid University, Abha 61413, Saudi Arabia, for funding this work through research groups program under grant number R.G.P.1/30/42.

\section{References}

[1] R. Hilfer, Ed., Applications of fractional calculus in physics, World Scientific, 2000.

[2] A. A. Kilbas, H. M. Srivastava, and J. J. Trujillo, Theory and Applications of Fractional Differential Equations. Vol. 204, Elsevier, 2006.

[3] S. Kumar, "A new fractional modeling arising in engineering sciences and its analytical approximate solution," Alexandria Engineering Journal, vol. 52, no. 4, pp. 813-819, 2013.

[4] A. Yildirim, "Application of He's homotopy perturbation method for solving the Cauchy reaction-diffusion problem," Computers \& Mathematics with Applications, vol. 57, no. 4, pp. 612-618, 2009. 
[5] S. Ali, S. Bushnaq, K. Shah, and M. Arif, "Numerical treatment of fractional order Cauchy reaction diffusion equations," Chaos, Solitons \& Fractals, vol. 103, pp. 578-587, 2017.

[6] N. F. Britton, Reaction-Diffusion Equations and Their Applications to Biology, Academic Press, 1986.

[7] R. S. Cantrell and C. Cosner, Spatial ecology via reactiondiffusion equations, John Wiley \& Sons, 2004.

[8] P. Grindrod, The Theory and Applications of ReactionDiffusion Equations: Patterns and Waves, Clarendon Press, 1996.

[9] J. Smoller, Shock Waves and Reaction-Diffusion Equations. Vol. 258, Springer Science \& Business Media, 2012.

[10] S. S. Behzadi, "Solving Cauchy reaction-diffusion equation by using Picard method," SpringerPlus, vol. 2, no. 1, pp. 1-6, 2013.

[11] M. I. A. Othman and A. M. S. Mahdy, "Differential transformation method and variation iteration method for cauchy reaction-diffusion problems," Journal of Mathematics and Computer Science, vol. 1, no. 2, pp. 61-75, 2010.

[12] H. Rouhparvar, S. Abbasbandy, and T. Allahviranloo, "Existence and uniqueness of solution of an uncertain characteristic cauchy reaction-diffusion equation by Adomian decomposition method," Mathematical and Computational Applications, vol. 15, no. 3, pp. 404-419, 2010.

[13] S. Das, R. Kumar, and P. K. Gupta, "The homotopy analysis method for fractional Cauchy reaction-diffusion problems," International Journal of Chemical Reactor Engineering, vol. 9, no. $1,2011$.

[14] H. Ahmad, T. A. Khan, I. Ahmad, P. S. Stanimirovic, and Y.-M. Chu, "A new analyzing technique for nonlinear time fractional Cauchy reaction- diffusion model equations," Results in Physics, vol. 19, article 103462, 2020.

[15] K. Wang and S. Liu, "A new Sumudu transform iterative method for time-fractional Cauchy reaction-diffusion equation," SpringerPlus, vol. 5, no. 1, pp. 1-20, 2016.

[16] O. A. Arqub, R. Edwan, M. Al-Smadi, and S. Momani, "Solving space-fractional Cauchy problem by modified finite-difference discretization scheme," Alexandria Engineering Journal, vol. 59, no. 4, pp. 2409-2417, 2020.

[17] V. Daftardar-Gejji and H. Jafari, "An iterative method for solving nonlinear functional equations," Journal of Mathematical Analysis and Applications, vol. 316, no. 2, pp. 753-763, 2006.

[18] M. Safari, D. D. Ganji, and M. Moslemi, “Application of He's variational iteration method and Adomian's decomposition method to the fractional KdV-Burgers-Kuramoto equation," Computers \& Mathematics with Applications, vol. 58, no. 11-12, pp. 2091-2097, 2009.

[19] G. Adomian, "A review of the decomposition method in applied mathematics," Journal of Mathematical Analysis and Applications, vol. 135, no. 2, pp. 501-544, 1988.

[20] A.-M. Wazwaz, "A reliable modification of Adomian decomposition method," Applied Mathematics and Computation, vol. 102, no. 1, pp. 77-86, 1999.

[21] S. Maitama and W. Zhao, "Homotopy perturbation Shehu transform method for solving fractional models arising in applied sciences," Journal of Applied Mathematics and Computational Mechanics, vol. 20, no. 1, pp. 71-82, 2021.

[22] S. Gupta, D. Kumar, and J. Singh, "Analytical solutions of convection-diffusion problems by combining Laplace transform method and homotopy perturbation method," Alexandria Engineering Journal, vol. 54, no. 3, pp. 645-651, 2015.
[23] J. Singh, D. Kumar, and S. Rathore, "Application of homotopy perturbation transform method for solving linear and nonlinear Klein-Gordon equations," Journal of Information and Computing Science, vol. 7, no. 2, pp. 131-139, 2012.

[24] J. A. T. Machado, D. Baleanu, W. Chen, and J. Sabatier, "New trends in fractional dynamics," Journal of Vibration and Control, vol. 20, no. 7, pp. 963-963, 2014.

[25] D. Baleanu, Z. B. Güvenç, and J. A. Tenreiro Machado, Eds., New Trends in Nanotechnology and Fractional Calculus Applications, Springer, New York, NY, USA, 2010.

[26] S. Maitama and W. Zhao, "New integral transform: Shehu transform a generalization of Sumudu and Laplace transform for solving differential equations," 2019, http://arxiv.org/abs/ 1904.11370.

[27] A. Bokhari, "Application of Shehu transform to AtanganaBaleanu derivatives," Journal of Mathematics and Computer Science, vol. 20, no. 2, pp. 101-107, 2019.

[28] R. Belgacem, D. Baleanu, and A. Bokhari, "Shehu transform and applications to Caputo-fractional differential equations," International Journal of Analysis and Applications, vol. 17, no. 6, pp. 917-927, 2019. 